\title{
Analytical Processing of Applied Tasks Conceptual Models at Design of Information-Active Systems
}

\author{
Olga Novoselova ${ }^{1, *}$, Anton Ruzheynikov ${ }^{1}$ and Andrey Gavrilov ${ }^{1}$ \\ ${ }^{1}$ Department of information technologies and computing systems, Institute of information systems and technologies, Moscow State \\ Technological University "STANKIN", RU-127055, Moscow, Russia
}

\begin{abstract}
The article describes the concept of the methodology of intellectual labor automation, a formal description of the conceptual model (CM) at the object level, and also a description of the analytical processing of CM. The conceptual model is the basis for the design of the system. The conceptual model of an applied task reflects the semantics (the system of knowledge) of the subject field in the form of static and dynamic structures and their linkage. The elements of the static structure are subject categories defined by classes: cycle, process, task, component, object, feature, value. The dynamic structure reflects the system of subject dependences which describes the process of performing a subject task. The main stage of conceptual model analytical processing is the restructuring of the dynamic component. The method and technique of descriptions restructuring of subject dependences system at the level of features are developed. The method allows to provide reduction of time and spatial expenses on performance of process of the decision of the applied task in the computing environment at early stages of information-active systems design
\end{abstract}

\section{Introduction}

At automation of subject tasks, it is necessary to execute modeling of task decision process and a system design automated, information, information-active, intellectual, etc. Now there are many methods and methodologies allowing to form models of tasks and projects of systems. One of them is the methodology automation of intellectual labor (MAIL).

The methodology of intellectual labor automation [12] developed at the Moscow State University of Technology "STANKIN" offers an industrial method of automated systems creation and determines a necessary and sufficient set of model representations. These representations allow to present both the task performance process and the project of the automated and information-active system. The set includes the following descriptions: a) a subject task performance process (vision by an expert) - initial model representation [2, 3]; b) a knowledge system structure about subject field - conceptual model representation; c) an automated system project consisted of two model representations: infological and datalogical - invariant to the program and technical environment and implementers and one oriented to them.

According to MAIL, the system design process includes the following stages: initial, conceptual and infological modeling. Schematically, the methodology of intellectual labor automation (MAIL) is presented in figure 1.

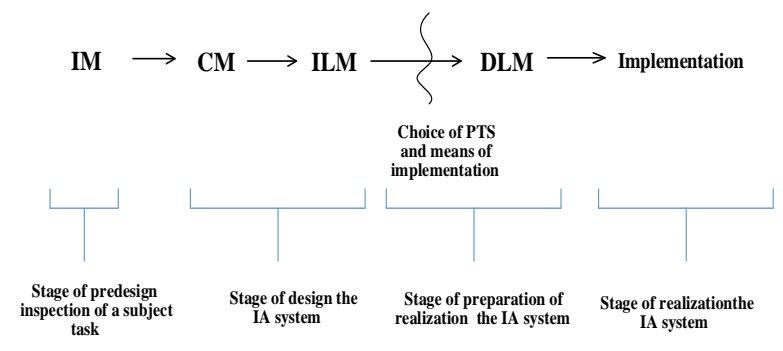

Fig.1. Methodology of automation of intellectual work, where IM is the initial model, CM - conceptual model, ILM infological model, DLM - datalogical model, PTS - software and hardware environment

Each model includes the description of informational or static, functional or/and dynamic components, a model in general. Informational (or static) component describes static elements structure of model. A functional component is an algorithm of the operations performed within a subject task. A dynamic component is structure of restrictions for static structure and accesses to it. The model in general defines coordination of functional or/and dynamic and informational or static components. At the same time there are components: for an initial model - functional and informational, for a conceptual model - dynamic and static, for an infological model - functional, dynamic and static.

The conceptual model is the basis for the design of the system. The conceptual model applied task reflects the semantics (the system of knowledge) of the subject field in the form of static and dynamic structures and their linkage. 
The description for each one of the components is formed as a special diagrams and specifications.

\section{Conceptual model (CM)}

The conceptual model of the applied task provides semantic unity of all subsequent models of this task [4, 5]. The static structure of the conceptual model describes the system of concepts of the subject field and consists of elements, derivative structures - schemes, also binary and ternary relations established between elements and between schemes. Conceptual structure formation is carried out at seven levels. Levels define classes of subject categories - cycle, process, task, component, object, feature, value.

The dynamic structure is a decomposition of the task into subtasks and includes descriptions of subject dependencies system of the 1st and 2nd kind. The subject dependences system of the 1st kind defines restrictions - interrelation of subject categories of the class "feature". The subject dependencies system of the 2nd kind defines restrictions on the same subject categories taking into account their "family tree" in the conceptual structure - the structure of arrangement in schemes.

The description of dynamic and static structures in the formation of the conceptual model is performed in graphical form (diagrams) and in tabular form (specifications).

Visual notation includes a set of elements (blocks and arrows); structures of these elements: sequence, iteration, alternative, cycle, switch; rules of charting and models. The composition and structure of the diagrams is fixed in the specifications. The 1st kind of subject dependencies system denotes the use of information only from the level of the conceptual structure of the type "feature" to perform actions (subject dependencies) while solving the task.

The conceptual model of the object level for the n-th applied task can be formally described as a set of structures and their linkage:

$$
K P 2(n)=<M 2(n), T H 2(n), \overline{F U 2}(n), R_{2}^{K P}(n)>.
$$

$M 2(n)$ - multitude of subject categories.

$$
M 2(n) \equiv A(n)=\left\{a_{k m}\right\} .
$$

Subject categories are divided into classes. They are formed in accordance with 7 semantic levels: cycle, process, task, component, object, feature, value. In this case, each feature is a property of an object.

$$
T H 2(n)=<T 2(n), H 2(n), \overline{H 2}(n), T h 2(n)>,
$$

where $T 2(n)$ - multitude of binary relations, $H 2(n)$ - multitude of ternary relations on subject categories,

$\overline{H 2}(n)$ - multitude of schemes of subject categories, $\operatorname{Th2}(n)$ - multitude of binary relations on schemes of subject categories.

$$
T 2(n) \subset A(n) \times A(n) .
$$

Multitude $H 2(n)$ can be represented as a set of subsets of a certain kind (see fig.2).

$$
H 2(n)=\left\{H A_{i}(n) A_{j}(n) A_{k}(n)\right\} .
$$

Under the scheme of subject categories for subject dependencies we will understand the multitude of elementary schemes defined by:

- general (one) contextual subject category;

- non-repetitive structural subject categories.

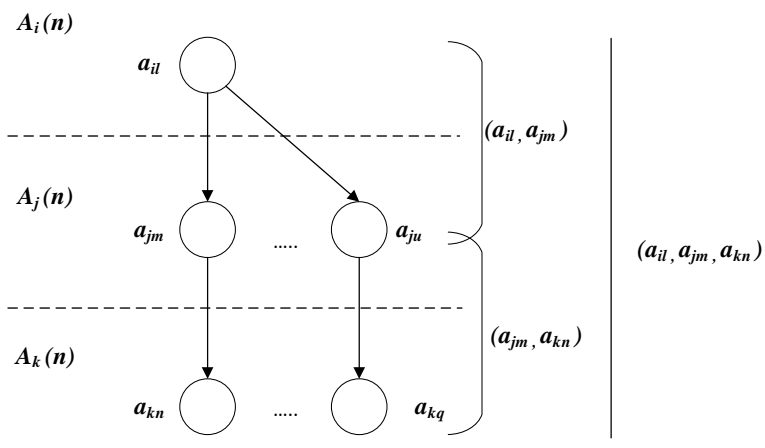

Fig. 2. Binary and ternary connections of subject categories

Formally, this is represented in the form

$$
\overline{h^{2}{ }_{l l}}=\left\{h_{\text {iljmkn }}^{2}, \ldots, h_{\text {iljukq }}^{2}\right\}, \quad m \neq u .
$$

Then the multitude of schemes of subject categories or derived constructions is defined for the subject task:

$$
\overline{H 2}(n)=\left\{\overline{h_{l l}^{2}}\right\} .
$$

Binary relations can be established on schemes of subject categories:

$$
\operatorname{Th} 2(n) \subset \overline{H 2}(n) \times \overline{H 2}(n) .
$$

Multitude $\operatorname{Th2}(n)$ can be represented as a set of subsets of a certain kind:

$$
\begin{gathered}
\operatorname{Th} 2(n)= \\
=\left\{L \bar{H} 2_{i}(n), L \bar{H} 2_{i}(n) \bar{H} 2_{k}(n), L \bar{H} 2_{k}(n) / L \bar{H} 2_{i}(n)\right\} .
\end{gathered}
$$

Binary relationships in category schemes reflect the connectivity of semantic complexity levels or "semantic scaling".

The dynamic relations $\overline{F U 2}(n)$ include two kinds of constraints - subject dependencies systems of the first $\overline{F 2}(n)$ and second $\overline{U 2}(n)$ kind:

$$
\begin{aligned}
\overline{\mathrm{FU} 2}(\mathrm{n}) & =\langle\overline{\mathrm{F} 2}(\mathrm{n}), \overline{\mathrm{U} 2}(\mathrm{n})\rangle . \\
\overline{\mathrm{F} 2}(\mathrm{n}) & =(\overline{\mathrm{V} 2}(\mathrm{n}), \overline{\mathrm{FV} 2}(\mathrm{n})),
\end{aligned}
$$

where $\overline{V 2}(n)$ - the multitude of subject dependencies of the 1 st kind and $\overline{F V 2}(n)$ - multitude of binary relations between subject dependencies of the 1 st kind. 


$$
\begin{gathered}
\overline{\mathrm{V} 2}(\mathrm{n}) \equiv \overline{\mathrm{W}}(\mathrm{n}), \\
\overline{\mathrm{W}}(\mathrm{n})=\bigcup_{\mathrm{p}} \overline{\mathrm{W}_{\mathrm{p}}}(\mathrm{n}), \\
\overline{\mathrm{W}_{\mathrm{p}}}(\mathrm{n})=\left\{\overline{\mathrm{w}_{\mathrm{ps}}}\right\} .
\end{gathered}
$$

The multitude of subject dependencies is divided into subsets of different levels decomposition:

$$
\begin{gathered}
\overline{\mathrm{FV} 2}(\mathrm{n}) \subset \overline{\mathrm{W}}(\mathrm{n}) \times \overline{\mathrm{W}}(\mathrm{n}), \\
\overline{\mathrm{FV} 2}(\mathrm{n})= \\
=\left\{\mathrm{L} \overline{\mathrm{W}_{\mathrm{p}}}(\mathrm{n}), \mathrm{L} \overline{\mathrm{W}_{\mathrm{p}}}(\mathrm{n}) \overline{\mathrm{W}_{\mathrm{t}}}(\mathrm{n}), \mathrm{L} \overline{\mathrm{W}_{\mathrm{t}}}(\mathrm{n}) / \overline{\mathrm{W}_{\mathrm{p}}}(\mathrm{n})\right\} .
\end{gathered}
$$

A submultitude $L \overline{W_{p}}(n)$ define binary relations as type "one after another" between subject dependencies of the 1st kind of one level.

A submultitude $L \overline{W_{p}}(n) \overline{W_{t}}(n)$ define binary relations as type "composition" between the object dependencies of the 1st kind of different levels.

A submultitude $L \overline{W_{t}}(n) / \overline{W_{p}}(n)$ define the binary relations as type "arrangement" between the subject dependencies of the 1st kind of the one level.

Since subject dependencies are considered at the object level, any dependency of the 1st kind is a variant of a certain type always. Since the type of dependence is fixed on a certain multitude of categories always, the subject dependence of the 1st kind is fixed on a subset of this categories multitude:

$$
\overline{w_{p s}}=\overline{w_{p s}}\left(i, t, u_{p s}\right) .
$$

The relations describing interrelations of the basic static and dynamic components of applied task model of object level can be represented in the following form:

$$
\begin{gathered}
\mathrm{R}_{2}^{\mathrm{KP}-1}(\mathrm{n}) \subset \mathrm{A}(\mathrm{n}) \times \mathrm{A}(\mathrm{n}) \ldots \times \mathrm{A}(\mathrm{n}) \times \overline{\mathrm{W}}(\mathrm{n}), \\
\mathrm{R}_{2}^{\mathrm{KP}-1}(\mathrm{n})= \\
=\left\{\mathrm{r}_{1}^{\mathrm{KP} 2-1} \mid \mathrm{r}_{1}^{\mathrm{KP} 2-1}:\left(\mathrm{a}_{\mathrm{j} 1}, \ldots, \mathrm{a}_{\mathrm{jq}}\right) \rightarrow \overline{\mathrm{w}_{\mathrm{ps}}}\right.
\end{gathered}
$$

\section{Analytical processing of the conceptual model}

For analytical processing of conceptual models it is possible to define the following complex of tasks: definition and check of information connectivity between subject dependences; analysis and restructuring of subject dependences system; fragmentation of information. [5, 6]

To analyze the system of subject dependencies on the possibility of its restructuring, it is necessary to obtain an information description (substantial features) for all subject dependencies - first elementary, then complex.
At the same time, the type of algorithmic construction should be taken into account for complex ones. The result should be a complete information description of the task on the whole (complex subject dependence of the top level), which is based on the content features description of all its constituent elementary (descendants) dependencies.

To transform the graphical descriptions, it was necessary to form a description of performing subject dependencies process in a different form. For this purpose, the "conveyor - time step" approach was proposed and the apparatus of block matrices was chosen. This apparatus allowed to present the system of dependencies in the form of a structural description [3, 5, 7]. The structural description takes into account the spatial-temporal characteristics of solving task process the axis of the conveyors $(\mathrm{K})$ is located vertically and the axis of the time steps $(\mathrm{T})$ is located horizontally.

Let's present a structural description $\overline{w_{k t}}$ - complex subject dependence in the form of a block matrix $B_{k t}$ :

$$
B_{k t}=\left[\begin{array}{ll}
B_{k t}^{1} & B_{k t}^{2} \\
B_{k t}^{4} & B_{k t}^{3}
\end{array}\right],
$$

where $B_{k t}^{1}$ - matrix-column defines the external (input) information connection $\overline{w_{k t}}$ complex subject dependence by input subject categories of the type feature";

$B_{k t}^{2}$ - square matrix determines the internal information relationship between the features of the subject dependencies $(k+1)$ of the decomposition level. These subject dependencies are part of the complex subject dependence $\overline{w_{k t}}$;

$B_{k t}^{3}$ - matrix-string defines an external (output) information connection $\overline{w_{k t}}$ complex subject dependence by output subject categories of the type "feature";

$B_{k t}^{4}$ - matrix-element. It shows what type of algorithmic construction the description belongs to: ' 1 ' sequence, ' $n$ ' - iteration, ' 0 ' - alternative.

The dimension of the matrix $B_{k t}$ is composed of sizes its constituent block matrices $B_{k t}^{1}, B_{k t}^{2}, B_{k t}^{3}, B_{k t}^{4}$ and dimension matrix is $m_{k t}=(m+1) \times(m+1)$.

Next, to form a structural description for the task, structural descriptions are created for all complex subject dependencies, which are then iteratively, starting from the top level, included in the matrix describing the task as a whole. At the same time, the elements of the matrix should be specified. These elements are showing the information connectivity of subject dependencies at this level of decomposition. At each step, the dimension of the resulting matrix $B_{k t}^{*}$ includes dimensions $B_{k t}$ (base matrix) and $B_{(k+1) t}$ (embedded matrix). The result is a matrix of the complete structural description of the task, where the horizontal shows the time spent on the task time steps, and the vertical spatial chains - conveyors.

An extended structural description $\overline{B_{k t}^{*}}$ is formed on the basis of the full structural description. This matrix is obtained by transposing the elements of the matrix $B_{k t}^{2 *}$ relative to the main diagonal. [8]

Then, after building a complete structural description of $B_{k t}^{*}$ and extended $\overline{B_{k t}^{*}}$, you can perform the transformation of the descriptions of subject 
dependencies system. To simplify the formal description, the block matrix $\overline{B_{k t}^{*}}$ is copied to the block matrix $C_{k t}$ :

$$
\begin{aligned}
& \overline{\mathrm{B}_{\mathrm{kt}}^{*}} \equiv \mathrm{C}_{\mathrm{kt}} \text {. } \\
& \mathrm{C}_{\mathrm{kt}}=\left[\begin{array}{cc}
\mathrm{C}_{\mathrm{kt}}^{1} & \mathrm{C}_{\mathrm{kt}}^{2} \\
\mathrm{C}_{\mathrm{kt}}^{4} & \mathrm{C}_{\mathrm{kt}}^{3}
\end{array}\right]= \\
& {\left[\begin{array}{cccccc}
\left(c_{10}^{p}\right)^{1-k t} & \left(c_{11}^{p}\right)^{2-k t} & \ldots & \left(c_{1 s}^{p}\right)^{2-k t} & \ldots & \left(c_{1 m}^{p}\right)^{2-k t} \\
\vdots & \vdots & \ddots & \vdots & \ddots & \vdots \\
\left(c_{s 0}^{p}\right)^{1-k t} & \left(c_{s 1}^{p}\right)^{2-k t} & \ldots & \left(c_{s s}^{p}\right)^{2-k t} & \ldots & \left(c_{s m}^{p}\right)^{2-k t} \\
\vdots & \vdots & \ddots & \vdots & \ddots & \vdots \\
\left(c_{m 0}^{p}\right)^{1-k t} & \left(c_{m 1}^{p}\right)^{2-k t} & \ldots & \left(c_{m s}^{p}\right)^{2-k t} & \ldots & \left(c_{m m}^{p}\right)^{2-k t} \\
\left(c_{(m+1) 0}^{p}\right)^{4-k t} & \left(c_{(m+1) 1}^{p}\right)^{3-k t} & \ldots & \left(c_{(m+1) s}^{p}\right)^{3-k t} & \ldots & \left(c_{(m+1)(m+1)}^{p}\right)^{3-k t}
\end{array}\right]}
\end{aligned}
$$

As a result of the analysis of the received structural description it is possible to carry out restructuring of subject dependences system which actually is algorithm optimization process of applied task decision on criteria of time and spatial expenses [6, 9, 10, 11]. Determining the time characteristic (number of time steps) is to find the maximum number of time step in the optimized structural description of the task. Determining the spatial characteristic (number of conveyors) is to find the maximum number of the conveyor in the optimized structural description of the task.

In order to obtain an optimal structural description over time steps, we formally described the motion of the main diagonal element in the matrix-block $C_{k t}^{2}$. The subject dependence (the main diagonal element) and all its associated elements moves to a time step earlier. You can move items only if there is no information connectivity between subject dependences. The absence or presence of information connectivity shows between subject dependencies the value " 0 " or " 1 " in the lower triangular matrix under the main diagonal.

The elements are moved according to the movement of the main diagonal elements in the $C_{k t}^{3}$. block. Blocks $C_{k t}^{1}, C_{k t}^{4}$ block matrix $C_{k t}$. remain unchanged.

As a result of the restructuring of subject dependences system 1st kind, we receive an optimal structural description according to the time characteristic in the form of a block matrix $C_{k t}$, which contains a horizontally compressed structural description of the matrix $\overline{B_{k t}^{*}}[3,4]$.

The block matrix $C_{k t}$ is copied to the block matrix $D_{k t}$ to simplify the restructuring of subject dependencies system of the 1st kind by spatial characteristic:

$$
C_{k t} \equiv D_{k t}
$$

To obtain the optimal structural description on the conveyors, the element of the main diagonal (the subject dependence) is moved to another conveyor located above, provided that, there is no information connectivity. Together with this element (subject dependency), all elements from this conveyor are moved. The move process is iteratively.

To move, the last element (on the main diagonal on the last conveyor (in the last row) of the $\mathrm{D}_{\mathrm{kt}}^{2}$ matrix) is defined. Next, we determine the element preceding it-the subject dependence. We need check the presence (1) / absence (0) of information connectivity between them. If there is no information connectivity, we can perform the move. The move rules are similar to the move rules when restructuring the structural description by time steps, only carry out movement from the line to the line above.

During the restructuring process, the main conveyor are formed of subject dependencies chain of the maximum length and additional conveyors to the main one. Typically, these conveyors contain subject dependencies of the type "alternative" as part of given information-related dependencies chain. Blocks $\mathrm{D}_{\mathrm{kt}}^{3}, \mathrm{D}_{\mathrm{kt}}^{4}$ block matrix $\mathrm{D}_{\mathrm{kt}}$ remain unchanged.

As a result of these actions, a block matrix $D_{k t}$, is obtained. This matrix contain a vertically compressed structural description of the matrix $\mathrm{C}_{\mathrm{kt}}$.

Fragmentation of information shows the distribution of subject dependencies of the task and their characteristics on performance time steps.

\section{Conclusion}

Thus, the analytical processing of subject dependencies system performs a set of tasks. The main from these tasks is the restructuring of subject dependencies system. The proposed conveyor-time steps approach and rules of formation and restructuring (optimization) of subject dependencies structural description allowed to parallelize the dynamic structure of the task taking into account the information connectivity of the dependencies. As result of restructuring (optimization) of subject dependencies system by time steps and conveyors, the task execution time is reasonably reduced, and the sequential process of solving the problem is transformed into a series-parallel one.

This research was supported by the project No. 17-29-07057 "Development of methods of restructuring and integration for semantic and syntactic representations when creating automation systems for design and management processes" of the RFBR. We also thank the Center of Collective Use of MSUT "STANKIN" for providing resources.

\section{References}

1. G.D. Volkova, Methodology automation of intellectual labor. Monograph (Moscow: Janus-K, 2013)

2. O.V. Novoselova, G.D. Volkova and Y.M. Solomentsev, Nonlinearity: Problems, Solutions and Application 2, 223-238 (New York, NY: Nova Science Publishers, Inc., 2017)

3. G.D. Volkova, S.M. Kuryshev, Automation and control in mechanical engineering 7 (1999)

4. G.D. Volkova, Intersectoral information service 3(148), 10-23 (2009)

5. M.V. Shchukin, Materials of V-th scientific conference of MSTU "STANKIN" and "Educational 
and scientific center of mathematical modeling of MSTU "STANKIN" - IMM RAS", 61-65 (2002)

6. O.V. Novoselova, A.A. Ruzheynikov, Information technologies in modeling and management: approaches, methods, solutions: Collection of scientific articles of the II all-Russian scientific conference with international participation 1, 511516 (2019)

7. F.R. Gantmakher, Theory of matrices (Moscow: Fizmatlit, 2004)

8. G.D. Volkova, N.M. Efromeev, Computer science and information technology: proceedings of the international scientific conference, 80-84 (2012)

9. O.V. Novoselova, N.V. Solodovnikova, Proceedings of higher educational institutions. Volga region. Technical science. Scientific and practical journal 1(49), 34-40 (2019)

10. N.V. Solodovnikova, Bulletin of modern research. Electronic scientific and practical periodical 1-3(28), 207-212 (2019)

11. O.V. Novoselova, O.V. Lyubchikova, Cutting-edge science - 2015: Materials of the XI International scientific and practical conference 27, 49-52 (2015) 